\title{
Objetos de aprendizagem da área de Geografia: relatos da experiência de desenvolvimento do Capitão \\ Tormenta e Paco em movimentos da terra, rede geográfica, fusos horários e estações do ano.
}

\author{
Meri Lourdes Bezzi - Universidade Federal de Santa Maria - meri@oslo.ccne.ufsm.br \\ Ana Claudia Giordani - Universidade Federal de Santa Maria - geo_giordani@yahoo.com.br \\ Roberto Cassol - Universidade Federal de Santa Maria - rtocassol@gmail.com \\ Ana Claudia Pavão Siluk - Universidade Federal de Santa Maria - ana@pontotec.com.br \\ Carlos Gustavo Martins Hoelzel - Universidade Federal de Santa Maria - \\ cghoelzel@yahoo.com.br \\ André krusser Damalzzo - Universidade Federal de Santa Maria - \\ andredalmazo@pop.com.br \\ Eunice Maria Mussoi - Universidade Federal de Santa Maria - \\ emmussoi@yahoo.com.br
}

Resumo: O artigo descreve o processo de desenvolvimento dos objetos de aprendizagem de Geografia, que compõem a série denominada Capitão Tormenta e Paco. A série é composta por quatro objetos que contemplam os conteúdos de Fusos Horários, Movimentos da Terra, Redes Geográficas e Estações do Ano. O artigo descreve as fases de desenvolvimento dos objetos, referindo-se à metodologia RIVED, à linha pedagógica adotada e às fases de desenvolvimento ao mesmo tempo em que vai apresentando as interfaces dos objetos. O artigo é finalizado apontando os ganhos da equipe com o desenvolvimento e o atendimento às recomendações para efetivar a qualidade e usabilidades dos objetos de aprendizagem desenvolvidos.

Palavras Chave: Objetos de Aprendizagem, Metodologias de desenvolvimento, Geografia.

Learning of objects of the area of Geography: stories to of the experience of development of Captain Tormenta and Paco in movements of the land, geographic net, hourly spindles and stations of the year.

Abstract:. The article describes the development process of geography learning object, which make up the series called Captain Tormenta and Paco. The series is composed of four objects that contain the contents of Time Schedules, movements of the Earth, Geographical networks and Seasons of the Year. The article describes the stages of development of objects, referring to the methodology RIVED, the line adopted pedagogical and stages of development at the same time we will presenting the interfaces of the objects. The article is finalized pointing earnings of the team with the development and care of recommendations for effective the quality and usabilidades of learning object developed.

Key Word: Learning objects, methodologies of development, Geography.

\section{Introdução}

O uso crescente de recursos didáticos que promovem a aprendizagem vem impondo transformações nos ambientes educacionais, devido à evolução e desenvolvimento tecnológico. Essas mudanças afetam diretamente a forma como o professor utiliza os materiais didáticos de apoio a suas aulas, desenvolvendo ele próprio 
ou reutilizando os materiais já desenvolvidos. Do mesmo modo, modifica a forma como os alunos aprendem, colocando-os em contato com materiais pedagógicos que têm possibilidades diversas de manuseio e interação.

Neste sentido, os objetos de aprendizagem constituem ferramentas dinâmicas, interativas e lúdicas para auxiliar a prática docente, mediante o emprego de ferramentas que tornem o processo de ensino e aprendizagem mais prazeroso. Além do mais, através da capacidade de animação e simulação, os objetos de aprendizagem podem tornar mais simples a compreensão de fenômenos a serem estudados.

A construção de objetos educacionais, sobretudo os multimídias, demanda de tempo, conhecimento, recursos humanos e financeiros. Devido a essas características, a sua elaboração envolve uma equipe multidisciplinar, onde a especificidade de cada área confere a qualidade e usabilidade dos objetos. Os profissionais, especialistas em informática na educação, em design e na área do conhecimento apresentada, devem interagir de modo a colaborar com o processo de desenvolvimento dos objetos de aprendizagem.

Cabe ressaltar a importância da parceria entre as Instituições de Ensino Superior e a Rede Interativa Virtual de Educação (RIVED) para a produção dos objetos, pois se aperfeiçoam os recursos para a inserção da tecnologia na educação, auxiliando a formação de novos professores, profissionais da área da informática, do design e ainda, o desenvolvimento de metodologias inovadoras de aprendizagem. Neste sentido, a Universidade Federal de Santa Maria (UFSM) representa uma das universidades selecionadas para participar do Projeto RIVED, tendo uma equipe composta de professores e alunos, que foram devidamente capacitados para a produção de conteúdos educacionais digitais, através do curso Fabrica Virtual, ministrado pela equipe do RIVED/SEED.

Assim, esse artigo apresenta a experiência de desenvolvimento de objetos de aprendizagem na área de Geografia, realizada pela equipe multidisciplinar da UFSM. O objetivo do artigo e relatar o processo de desenvolvimento, desde a fase inicial até a finalização dos objetos de aprendizagem. Para tal, descreve a utilização da metodologia RIVED, linha pedagógica adotada e a descrição das fases de desenvolvimento.

\section{Os conceitos envolvidos}

Ao ser iniciado o desenvolvimento dos objetos de aprendizagem, a maioria dos profissionais envolvidos já haviam realizado o curso oferecido pela SEED, sobre desenvolvimento de objetos de aprendizagem. No entanto, a inexperiência de todos fez com que fossem realizados estudos sobre os termos e os conceitos que estão envolvidos com a temática.

Dessa forma, verificou-se que o termo objetos de aprendizagem ou objetos educacionais tem encontrado diversos sinônimos na literatura atual. Segundo a Wikipédia (2006), "Existem autores que os nomeiam de componentes de software educacional; conteúdos de objetos compartilháveis (ADL, 2001); objetos de conhecimento (MERRIL, 2001); objetos educacionais (SPHORER, 2001); e objetos de aprendizagem (IEEE/LTSC, 2000)". Nos estudos realizados, o termo que se encontra com maior incidência se refere a objetos de aprendizagem ou objetos educacionais.

O conceito aplicado ao termo objetos de aprendizagem define, segundo Tarouco (2003a), qualquer recurso suplementar ao processo de aprendizagem, que pode ser reusado para apoiar a aprendizagem e, geralmente, aplica-se a materiais educacionais projetados e construídos em pequenos conjuntos, com a finalidade de maximizar as situações de aprendizagem onde o objeto possa ser aplicado. 
De acordo com o Learning Objects Metadata Workgroup (apud WIKIPÉDIA, 2006), objetos de aprendizagem (Learning Objects) podem ser definidos por "qualquer entidade, digital ou não digital, que possa ser utilizada, reutilizada ou referenciada durante o aprendizado suportado por tecnologias". Assim, pode-se utilizar um objeto de aprendizagem em diferentes ambientes de aprendizagem, virtuais ou não.

A principal característica dos objetos de aprendizagem consiste na reutilização, ou seja, na possibilidade de ser utilizado por muitas pessoas e em ambientes diversos e dispersos. Essa característica é colocada em prática por meio de repositórios, que armazenam os objetos de forma lógica, permitindo sua localização com base em metadados, isto é, na sua categorização, que poderá ser por temas, por nível de dificuldade, por autor ou por relação com outros objetos. Os repositórios de objetos educacionais, então, se constituem, segundo Tarouco (2003a), em coleções de recursos de aprendizagem armazenados em bases de dados ou sistemas de arquivos. Tendo o entendimento dos termos e conceitos, a equipe multidisciplinar, partiu para o desenvolvimento do objeto de aprendizagem contando com professores e alunos de Geografia, com profissionais do design e da informática.

\section{Material e Métodos}

Os objetos desenvolvidos seguiram a metodologia de planejamento e desenvolvimento do RIVED, na qual os conteúdos abordados devem instigar a construção do conhecimento de forma crítica e criativa. Associado a isso, a metodologia RIVED prevê a utilização das potencialidades oferecidas pelas tecnologias da informação e comunicação, sobretudo informática, voltadas a abordagens pedagógicas inovadoras. Desse modo, a metodologia RIVED apresenta alguns padrões a serem seguidos, os quais auxiliam as equipes no desenvolvimento dos objetos, na medida em que propõe um modelo de design pedagógico, um roteiro para a construção, um Guia que auxilia o professor a melhor utilizar o objeto e um padrão visual, além de os objetos serem multiplataforma.

Apoiados na metodologia RIVED, buscou-se também uma linha pedagógica para o desenvolvimento dos objetos de aprendizagem multimídia, levando-se em consideração alguns requisitos importantes que o objeto deve contemplar, para que atenda às necessidades educativas e dos usuários.

Nessa mesma perspectiva, encontra-se em Valente (1998) a proposta de realização de um ciclo, quando se relaciona à construção e à aquisição de novos conhecimentos por parte do aluno, utilizando software educativo. Este ciclo se apresenta com as seguintes etapas: Descrição da resolução do problema; Execução dessa descrição pelo computador; Reflexão sobre o que foi produzido pelo computador $e$ Depuração dos conhecimentos por intermédio da busca de novas informações ou do pensar.

O processo de depuração dos conhecimentos acontece quando o aprendiz busca informações (conceitos, convenção de programação, etc.) em outros locais. Essas informações são então assimiladas pela estrutura mental, passando a ser conhecimento. Esse conhecimento será, por sua vez, utilizado no programa para modificar a descrição anteriormente definida. Nesse momento, repete-se o ciclo descrição - execução reflexão - depuração - descrição. Portanto, os objetos de aprendizagens desenvolvidos seguem o Design Pedagógico da metodologia RIVED, procurando atender às etapas propostas pelo Ciclo de aprendizagem de Valente (1998).

Em consonância com a linha pedagógica e a metodologia RIVED para o desenvolvimento de objetos de aprendizagem é necessário seguir alguns passos, que 
determinam a metodologia tecnológica adotada. Neste caso, foi utilizada a proposta de Bezerra (2002), que classifica as atividades a serem desenvolvidas em: levantamento de requisitos, análise, projeto, implementação, testes e implantação.

\section{Resultados e Discussão}

Para o desenvolvimento dos objetos de aprendizagem procurou-se atender à área de Geografia, a qual não estava contemplada no repositório do RIVED, naquele momento. A partir disso, se elegeu o conteúdo de fusos horários, pelo fato deste conteúdo apresentar baixo índice de acertos no programa do Concurso Vestibular e no Programa de Ingresso Ensino Superior (PEIES) da UFSM. Nesses processos seletivos, percebeu-se que o grau de dificuldade dos alunos é significativo, certamente por envolver cálculos e raciocínio lógico. Desse modo, o público alvo a ser atingido com o objeto é o ensino médio. Então, iniciou-se a fase de levantamento de requisitos.

Nesta fase, foram determinados quais os requisitos que o objeto deve ter. Para tanto, discutiu-se as funcionalidades do objeto a partir de estudo das necessidades dos usuários. Dentre as técnicas utilizadas, destaca-se o estudo teórico acerca da temática, entrevistas com especialistas na área de informática na educação e comparação com outros sistemas já existentes.

Segundo Bezerra (2002), o produto desta etapa é um documento, escrito em notação informal (linguagem natural), abordando:

a) requisitos funcionais, que delineiam as funcionalidades do objeto de aprendizagem (exemplo: o sistema deve permitir acesso aos professores e alunos mediante login e senha ou ao estar disponível é totalmente livre).

b) requisitos não-funcionais, que declaram as características do objeto em termos de confiabilidade (tolerância a falhas), desempenho (tempo de resposta esperado para as funcionalidades do sistema), portabilidade (restrições sobre plataformas de hardware e software, facilidade de transportar sistema para outras plataformas) e;

c) restrições em relação a custos, prazos, aspectos legais, plataforma tecnológica ou aquisição de novos softwares/equipamentos.

O levantamento dos requisitos necessários para a definição dos objetos propostos foi realizado a partir do estudo das áreas de conhecimento propostas nos objetos, nas perspectivas epistemológica e tecnológica, do levantamento sobre o "estado da arte" da área em questão, ressaltando, a partir da análise de alguns objetos de aprendizagem já existentes, e dos estudos sobre desenvolvimento e utilização de tecnologias de informação e comunicação na educação. Foram considerados também os estudos sobre as inovações no ensino das áreas em que estão sendo propostos os objetos.

Esta fase corresponde à elaboração do Design Pedagógico, que para a metodologia RIVED é um documento que descreve, em linhas gerais, as idéias dos autores para um determinado módulo. Ele traz os objetivos educacionais, o tema central e as atividades/estratégias de aprendizagem para o aluno. A elaboração do design pedagógico é a tarefa inicial no processo de desenvolvimento de um módulo educacional, e permite a equipe pedagógica fazer um esboço das atividades de ensino/aprendizagem.

Após estabelecer o que os alunos devem aprender, o próximo passo é formular um plano pedagógico para facilitar a aprendizagem dos alunos, como ensiná-los e quais combinações de canais de aprendizagem e tecnologias devem ser usadas. Tudo isso, levando-se em consideração as disponibilidades, a eficiência e o custo e benefício. 
A fase de análise e projeto permitiu que se estudasse o problema a ser apresentado no objeto, ou seja, o desenvolvimento do objeto proposto foi analisado, as fontes do problema foram identificadas e as possíveis soluções foram determinadas. Envolveu a definição de como alcançar os objetivos determinados durante a análise e expandir a fundamentação do objeto de aprendizagem.

Durante essa fase, o desenvolvimento começou a apresentar questionamentos, quando a equipe apresentou o conteúdo do objeto. Eram conteúdos extensos, alguns que necessitavam de outros conhecimentos e outros conteúdos que se co-relacionavam com os demais. O objeto fusos horários era sobrecarregado de textos e questões a serem atendidas. Embora apresentasse os conteúdos na forma de hipertexto, não continha aspectos intuitivos de navegabilidade. (Figura 1).

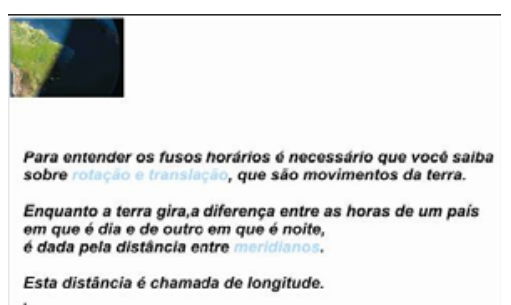

Figura 1: Proposta inicial do objeto de aprendizagem que envolvia conteúdos correlacionados.

Sobre essa questão, Sá filho; Machado dizem que no desenvolvimento de materiais didáticos é preciso pensar o conteúdo tratado em blocos pequenos. Assim, os objetos de aprendizagem tornam-se mais fáceis de serem reutilizados em contextos similares ou de serem modificados. A idéia é projetar cada bloco individualmente (com conteúdo independente), para que juntos, formem uma unidade maior. Logo, o propósito educacional de um objeto de aprendizagem é muito mais definido pelo uso do recurso que o desenvolvedor estabelece do que pelo recurso em si (SÁ FILHO; MACHADO).

Desse modo, teve-se o entendimento que deveriam ser quatro os objetos de aprendizagem, procurando privilegiar os quatro principais conceitos envolvidos no conteúdo de fusos horários, definidos pela equipe multidisciplinar. Assim, obtiveram-se Fusos horários, Movimentos da terra, Redes geográficas e Estações do ano.

A metáfora a ser utilizada nos objetos foi outro ponto que mereceu tempo de discussão da equipe. Iniciou-se pensando, ainda sobre fusos, em um casal de bailarinos, aproveitando os movimentos da dança (realizando a metáfora da terra girando em torno do sol). Após, evoluiu-se para alunos dançarinos de todos os gêneros musicais, aproveitando para dançarem em várias culturas, tempos históricos e estações do ano.

Avaliando-se essa metáfora, chegou-se a conclusão que, talvez, não fosse uma abordagem que trouxesse interesse aos alunos do Ensino Médio, do modo que estava sendo explorada. Por fim, entre tantas possibilidades discutidas, definiu-se por apresentar a metáfora da vida a bordo de um navio pirata, na qual há dois personagens, o Capitão Tormenta e o papagaio Paco, conforme demonstrado na Figura 2.

Com base na metáfora da história pirata, o Capitão Tormenta caracteriza-se por ser o velho capitão pirata, mal-humorado, mão-de-gancho e perna-de-pau. Já Paco, é o papagaio que sofre as sentenças impostas pelo capitão e auxilia o aluno no acesso ao conteúdo estudado. (Figura 2). 


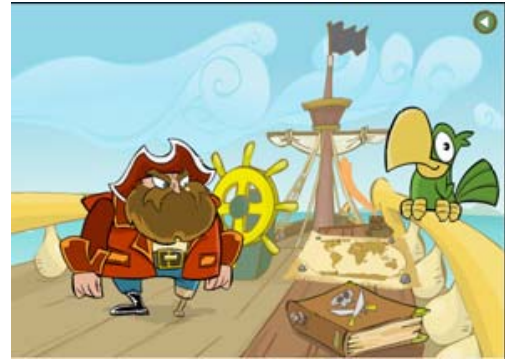

Figura 2: Metáfora e personagens Capitão Tormenta e Paco

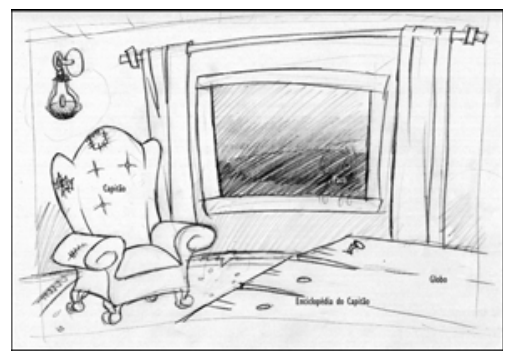

Figura 3: Desenvolvimento dos cenários

A composição dos personagens e a definição dos cenários, das ferramentas, do formato do texto, do áudio, do visual gráfico, da linguagem utilizada, das cores, do fluxo das informações e dos desafios que fazem parte dos objetos, estão presentes na fase de análise e projeto. Definidas essas questões, buscou-se desenvolver os story boards, fluxogramas, os personagens, as ilustrações e as animações, a figura 3 apresenta o desenvolvimento dos cenários.

Questões de caráter pedagógico que interferem em todos os objetos foram amplamente discutidas pela equipe multidisciplinar. Apoiados nos estudos realizados por Tarouco et al (2003b), observou-se o cuidado da sobrecarga cognitiva aplicada aos objetos educacionais. Esta refere-se às necessidades exigidas na memória de trabalho do aprendiz durante a instrução. Em se tratando de instrução baseada na web, refere-se tanto ao processo mental necessário para acessar e interpretar as telas, ícones e objetos, como o processo cognitivo dedicado para processar o real conteúdo da instrução (TAROUCO et al., 2003b).

Desse modo, os recursos utilizados no objeto de aprendizagem não devem superar, em níveis de dificuldades ou de motivação, o conteúdo proposto ou a tarefa solicitada.

O objetivo de desenvolver objetos educacionais é fazer com que o processo de aprendizagem do aluno ocorra de maneira mais eficaz possível. Para tanto, foi a partir das pesquisas de Lindermann (1983, apud TAROUCO, 2003b), que observou alunos utilizando material educacional online, combinando com o tempo entre a carga de uma etapa e a realização da seguinte, que foram feitas algumas recomendações sobre a apresentação de informações visando reduzir a carga cognitiva dos alunos que utilizam os materiais. Desse modo, as recomendações para apresentação de informações em projetos educacionais são: 1) simplicidade do texto, em qualidade e quantidade; 2) formatação confortável, a disposição do texto e das imagens devem estar em harmonia; 3) cores, que poderão ser utilizadas para atrair ou distrair o usuário; 4) segmentação, a disposição das informações não devem ocupar toda a tela do computador para evitar a monotonia na leitura; 5) uso de imagens e gráficos, para promover a aprendizagem de uma geração que vive em meio aos recursos visuais e de comunicação.

Procurando atender a essas recomendações, no que se refere à interação, como possibilidade de inovação e construção do conhecimento, os desafios impostos aos alunos são apresentados na forma de charadas a serem reveladas. Desse modo, o capitão convida o aluno a fazer parte da tripulação do seu navio, impondo-lhe desafios que devem ser superados. Assim, há a promoção da interação do aluno com o objeto e, conseqüentemente, do aluno com o conteúdo, pois passa a fazer parte da história que está acontecendo no navio, como ilustra a Figura 4. 


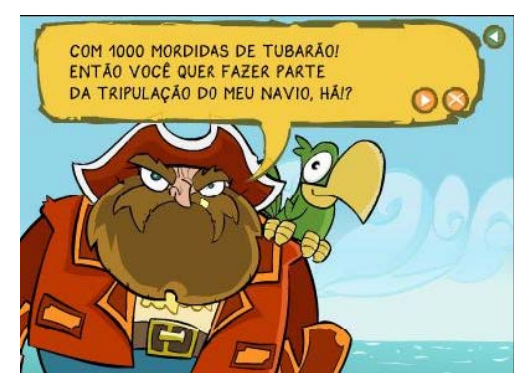

Figura 4- Capitão Tormenta e Paco em Movimentos da Terra

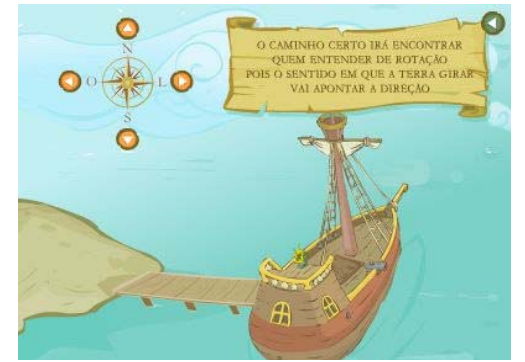

Figura 5- Apresentação da Charada

Essa interação leva à definição da linguagem e das ações que os personagens apresentam. Na Figura 5, pode-se observar que a charada apresentada pelo capitão utiliza rima como linguagem.

As charadas presentes nos objetos são uma das estratégias pedagógicas utilizadas, pois, apresentam o desafio de maneira lúdica, linguagem clara e texto simples de ser compreendido, não oferecendo sobrecarga cognitiva ao aluno.

Ainda referindo-se às estratégias pedagógicas e às recomendações sugeridas por Lindermann (1983, apud TAROUCO, 2003b), determinou-se função ao papagaio Paco, que é justamente mostrar ao aluno onde ele pode buscar as informações necessárias para revelar a charada. Paco aponta os links para os conteúdos, conforme pode ser visualizado na Figura 6.

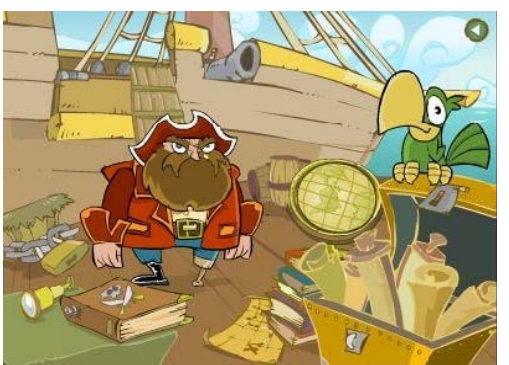

Figura 6- Elementos de interação

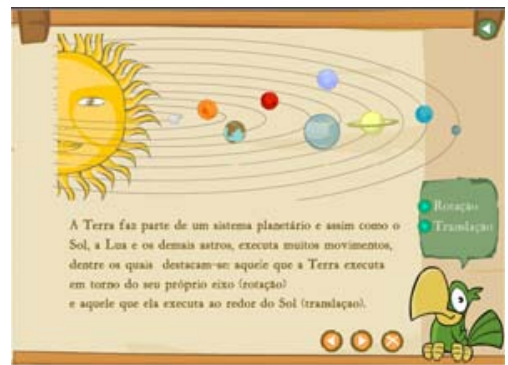

Figura 7: Texto de apoio

A partir da interface apresentada na Figura 6, o aluno pode acessar diferentes links. Destaca-se que a navegação depende da interação do usuário, ou seja, não há uma seqüência pré-estabelecida para o aluno seguir. Deste modo, acredita-se que o aluno terá seu raciocínio estimulado. Destaca-se os seguintes elementos da Figura 6:

A enciclopédia do Capitão: é um recurso com informações adicionais, tem a função de um "Saiba Mais".

O globo: clicando nele o aluno terá acesso ao conteúdo, com animações e simulações.

Paco: é o papagaio que serve para fornecer as instruções ao usuário.

Capitão Tormenta: clicando nele o aluno terá acesso a duas charadas, referentes ao conteúdo tratado.

Os textos presentes nos objetos são oferecidos como suporte ao conteúdo (Figura 7). Observa-se nesta figura que a interface que contém o texto, apresenta o mesmo design dos objetos. As interfaces parecem pergaminhos antigos e contêm poucos textos para facilitar a leitura dos alunos na tela. Nessa tela é possível verificar a presença de Paco, o qual se mostra durante todo o processo de navegação do os objetos, disponível para auxiliar o aluno com o conteúdo.

O tratamento dado ao acerto e erro do aluno está ligado às características inerentes a um velho capitão pirata. Desse modo, compreende-se que pelo fato dos objetos serem destinados aos alunos do ensino médio, que as ações do capitão nessas 
situações, serão naturalmente aceitas e entendidas, pois Paco e o aluno deixam de viajar com o capitão, cada vez que a charada não é revelada.

Assim, no objeto Fusos horários quando o aluno revela a charada, ele é comemorado pelo Capitão Tormenta , quando ele não revela, aparece Paco remando sobre as águas (Figura 8), o que significa que Paco não vai participar da tripulação.
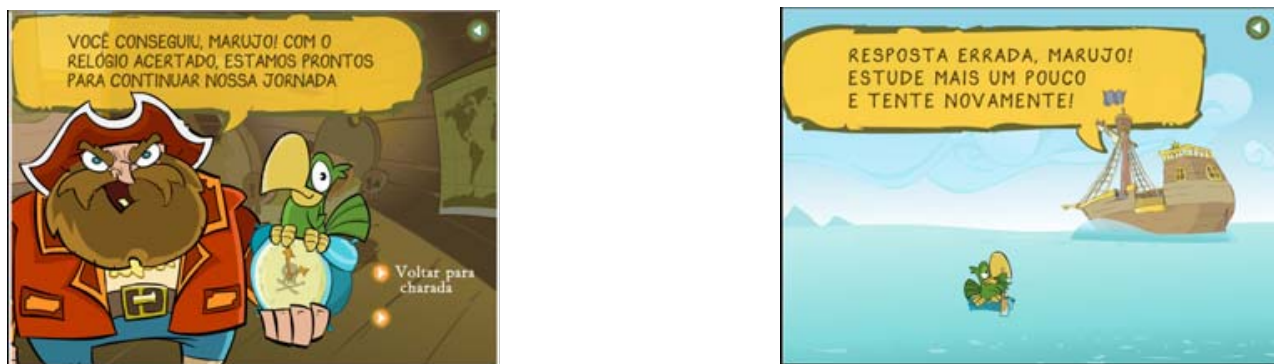

Figura 8- Capitão Tormenta e Paco em Fusos Horários- Resultado da charada.

No objeto Movimentos da terra, quando o aluno revela a charada acertando a direção que a terra deve girar, o marujo pode subir a borda para a aventura que os espera. Quando o aluno não coloca acerta as charadas ele é remetido a estudar mais e tentar novamente (Figura 9).
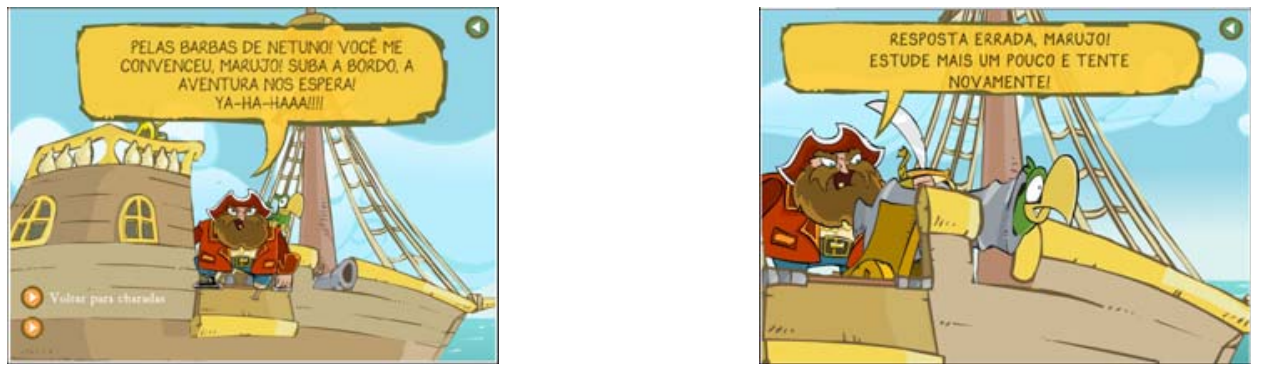

Figura 9 - Capitão Tormenta e Paco em Movimentos da Terra- Resultado da charada

Quanto à fase de implementação, essa teve como suporte as fases de análise e de projeto. O objetivo dessa etapa foi gerar materiais para auxiliar o desenvolvimento do objeto, bem como do seu design. Esta fase refere-se ao efetivo desenvolvimento do objeto, por meio da aplicação dos recursos tecnológicos identificados nas fases anteriores, utilizando-se para isso, o story board. Para a metodologia RIVED, nesta etapa, o grupo de técnicos produz os objetos de aprendizagem tendo como suporte a interação entre os especialistas de conteúdo, o designer instrucional e técnico, evitando, desse modo, falhas no desenvolvimento do objeto em termos técnicos e pedagógicos. Uma vez que os objetos foram desenvolvidos seguindo a metodologia RIVED, todos os padrões presentes na metodologia foram contemplados. Assim, no desenvolvimento do objeto de aprendizagem empregaram-se recursos de linguagem de programação, bem como os da Internet. A plataforma base do jogo é o programa Macromedia Flash.

Destaca-se que, atualmente, é o aplicativo mais utilizado para criações multimídia para a Internet. As causas para sua preferência são a facilidade de manipulação gráfica e a geração de arquivos de baixo tamanho e alta resolução. O padrão flash tem como propriedades uma animação vetorial em algumas aplicações, como seqüências de imagens e vídeo. Ocorre, portanto, o armazenamento apenas do vetor gerado, sendo que por meio deste vetor e de uma função geradora do processador gráfico do computador, que se cria todo o restante da imagem ou da animação. 
Após essa fase, ocorreram os testes e implantação que do objeto para entrar em funcionamento. A implantação consistiu em disponibilizar o objeto para que fosse utilizado, testando-o e fazendo-o funcionar adequadamente, segundo os parâmetros estabelecidos no projeto. Bezerra (2002) ao se reportar a etapa de testes no desenvolvimento de um sistema, destaca que apesar da existência de uma etapa específica para testagem, ela deve ocorrer ao longo do processo e, sobretudo, quando houver alterações no sistema. No caso dos objetos desenvolvidos, os testes foram sendo realizados na medida em que o objeto estava sendo desenvolvido. A última fase de desenvolvimento diz respeito à eficiência do objeto, ou seja, a avaliação.

Deve ocorrer ao longo de todo o processo - dentro das fases, entre as fases e após a implantação. A avaliação pode ser formativa ou somática. Avaliação formativa ocorre durante e entre as fases. Avaliação somática, em geral, ocorre após a versão final da implementação. Este tipo de avaliação verifica a eficiência do objeto de aprendizagem. Deste modo, primeiro realizou-se um estudo do projeto para a primeira avaliação, após ocorreu a segunda avaliação, onde procurou-se corrigir os erros encontrados e deixar o ambiente com características mais adequadas à demanda de seus usuários. Os objetos apresentados ainda estão em processo de validação, não tendo sido finalizada essa última etapa. Posteriormente a esta avaliação, será apresentado o objeto com as reformulações e, logo após, uma nova avaliação deverá ser realizada. Em se tratando de avaliação, a metodologia seguida pela Equipe multidisciplinar prevê a avaliação de usabilidade, que é definida como a capacidade que apresenta um sistema interativo de ser operado, de maneira eficaz, eficiente e agradável, em um determinado contexto de operação, para a realização das tarefas de seus usuários.

Desse modo, a avaliação de usabilidade de um sistema interativo tem como objetivos gerais: 1) validar a eficácia da interação humano-computador, face a efetiva realização das tarefas por parte dos usuários; 2) verificar a eficiência desta interação, face os recursos empregados (tempo, quantidade de incidentes, passos desnecessários, busca de ajuda, etc.) e 3) obter indícios da satisfação ou insatisfação (efeito subjetivo) que ela possa trazer ao usuário.

Estes objetivos devem ser pensados em relação aos diferentes contextos de operação previstos para os objetos. No caso dos objetos que compõem a série Capitão Tormenta e Paco, a validação está sendo realizada com alunos da Rede Pública Municipal, do município de Santa Maria, RS.

\section{CONCLUSÃO}

O desenvolvimento dos objetos de aprendizagem representa a construção de recursos didáticos para serem utilizados em sala de aula que têm o objetivo de estimular o senso crítico dos alunos bem como seu raciocínio servindo como um recurso que se integra no desenvolvimento curricular das escolas, com base em uma orientação pedagógica voltada para a possibilidade dos professores se tornarem mediadores do processo de ensino e aprendizado, junto com o conteúdo estudado e com o meio que apresenta ou representa o conteúdo.

Os objetos de aprendizagem, enquanto materiais pedagógicos, também possibilitam aos alunos, uma postura de aprendizes pensadores, investigadores e solucionadores de problemas, diante dos conteúdos apresentados. A disponibilização dos objetos em repositórios para reutilização, possibilita que outras pessoas os utilizem, otimizando os recursos já desenvolvidos, apresentando-se como mais uma vantagem de desenvolvimento de objetos. 
Quanto ao atendimento das recomendações acerca dos objetos de aprendizagem, embora tenham sido tomados alguns cuidados na sua construção, seguindo inclusive padrões estabelecidos mundialmente, observa-se que questões relacionadas à acessibilidade aos alunos com necessidades educacionais especiais, não foram contempladas. No entanto, os objetos que estão em desenvolvimento por essa equipe, já estão seguindo as recomendações internacionais sobre acessibilidade publicadas pelo consórcio W3C, fundador da WEB, que envolvem o Design Universal e desenvolvimento de ferramentas para os padrões WEB.

Os objetos de aprendizagem desenvolvidos pela equipe multidisciplinar da UFSM consistem em uma experiência inovadora para professores e instituição. Desse modo, reconhece-se que há inúmeras questões a serem revisadas e contempladas, ao final da fase de validação. No entanto, percebe-se que os professores participantes desse processo, assim como os alunos, já desenvolveram novas metodologias didáticas que utilizam recursos tecnológicos nas suas práticas. Quanto aos alunos, essa iniciação já obteve retorno, ao se conferir a premiação na $2^{\circ}$ e $3^{\circ}$ Edição do Concurso Rived, totalizando a premiação de sete objetos aprendizagem no Concurso RIVED dos anos de 2006 e 2007.

Portanto, por meio dos objetos de aprendizagem construídos e da apresentação de seu processo de desenvolvimento, busca-se contribuir para desenvolver nos alunos, habilidades para o pensamento criativo e reflexão crítica, e apresentar aos professores um recurso para modificar os métodos tradicionais de transmissão de informações, que enfatizam a repetição e a memorização passiva, por uma postura ativa de investigação e exploração de novas possibilidades.

\section{REFERÊNCIAS}

BEZERRA, Eduardo. Princípios de análise e projeto de sistemas com UML. Rio de Janeiro: Campus, 2002.

SÁ FILHO, Clovis Soares; MACHADO, Elian de Castro. O computador como agente transformador da educação e o papel do objeto de aprendizagem. Disponível em http://www.universia.com.br/materia/materia.jsp?id=5939 Acesso em 20 de jul. de 2006.

TAROUCO Liane M.; FABRE, Marie-Christine.; TAMUSIUNAS, Fabrício. Reusabilidade de objetos educacionais. 2003a. Disponível em http://www.cinted.ufrgs.br/renote/fev2003/artigos/marie_reusabilidade.pdf Acesso em 20 de jul. de 2006.

TAROUCO Liane M.; GRANDO, Anita; KONRATH, Mary Lúcia. Alfabetização visual para a produção de objetos educacionais. 2003b. Disponível em http://www.cinted.ufrgs.br/renote/set2003/artigos/artigo_anita.pdf Acesso em 20 de Jul. de 2006.

VALENTE, J. A. Análise dos diferentes tipos de softwares usados na educação. NIED- UNICAMP. Campinas: UNICAMP, 1998.

WIKIPÉDIA. Objetos de aprendizagem. 2006. Disponível em $<$ http://pt.wikipedia.org/wiki/Objetos_de_aprendizagem> Acesso em 10 de jun. de 2006. 